\title{
A COST-LOSS ANALYSIS TO EVALUATE THE VALUE OF WEATHER ESTIMATES AS INPUTS TO A DISEASE RISK MODEL
}

\author{
K.S. KIM, R.M. BERESFORD and W.R. HENSHALL \\ The Horticulture and Food Research Institute of New Zealand Limited, \\ Mt Albert Research Centre, Private Bag 92169, Auckland, New Zealand \\ Corresponding author: kkim@hortresearch.co.nz.
}

\begin{abstract}
To assess the value of weather estimates, the expenses, including the cost of spray application, and the losses incurred because of a disease were analysed using disease risk simulations. In this case study, the risk of botrytis bunch rot was simulated using weather estimates as inputs to a disease risk model. Those estimates were obtained using spatial interpolation and nearest neighbour methods. Possible cost was calculated based on 2 by 2 matrices under the assumption that fungicide spray decisions were made using the disease risk model with estimated weather data. The expenses associated with each combination of decisions on spray application and outcome were compared between weather estimates and measurements.
\end{abstract}

Keywords: cost-loss ratio, decision support system, botrytis bunch rot.

\section{INTRODUCTION}

Models have been developed to predict the risk of plant disease outbreaks and to provide information on the best course of action that a grower can take (Gleason 2000; Kim et al. 2006). Such models, which are a key component of a decision support system, quantify the risk of a disease outbreak under given weather conditions. Although weather data can be obtained from on-site weather stations, it is difficult to install and manage weather stations at every field, because of equipment and labour costs.

Weather data can be estimated using nearby weather stations for a site of interest (Meyers 1994). However, it is likely that weather estimates incur errors. As a result, a decision support system can give a false alarm, which is the non-occurrence of a predicted event. It is also possible that the system misses the occurrence of an event.

Errors of a system are often represented by the degree of disagreement between actual measurements and estimates. The same approach can be applied to analyse errors in predicting the risk of a disease using a decision support system (Kim et al. 2006). However, such an approach fails to indicate the economic implications of these errors, which are essential for growers to make a decision on disease management for their farm.

Richardson (2000) suggested a simple cost-loss model to evaluate the value of weather forecasts. The present study used the cost-loss model to determine the impact of errors in weather estimation on the value of a decision support system for managing the risk of a plant disease. The risk of botrytis bunch rot on grapes was simulated with a disease risk model using weather data at a specific site. Two methods for estimating weather data at that site (nearest neighbour method and spatial interpolation) were compared using a cost-loss model to determine their relative value.

\section{MATERIALS AND METHODS}

Richardson (2000) suggested a simple decision-analytic model using a two by two matrix. Suppose a decision maker has two choices: to take action or to do nothing. The outcome of the choice that a decision maker makes will be dependent on whether a 
given weather condition occurs or not. When no action has been taken but the weather event occurs, the decision maker suffers a loss $L$. If the decision maker takes action, e.g. spray application, an expense $X$ will be incurred irrespective of the outcome. If the event occurs, the action prevents part of the loss, which represents the potential gain $\mathrm{G}$. The cost (expense and loss) associated with each combination of action and occurrence of the weather event is listed in Table 1.

TABLE 1: Cost for actions that a decision maker may take.

\begin{tabular}{lcc}
\hline & \multicolumn{2}{c}{ Action } \\
\cline { 2 - 3 } Occurrence & Not taken & Taken \\
\hline No & 0 & $\mathrm{X}$ \\
Yes & $\mathrm{L}$ & $\mathrm{X}+\mathrm{L}-\mathrm{G}$ \\
\hline
\end{tabular}

Suppose the event is the occurrence of weather conditions favourable for botrytis bunch rot at a specific vineyard site. Weather measurements $M$ from an on-site weather station would allow the decision maker to assess the risk of botrytis bunch rot and to take action to minimise losses. Under such circumstances, the expected cost is as follows:

$$
E_{M}=\bar{o} \cdot(X+L-G),
$$

where $\overline{\boldsymbol{O}}$ is the fraction of occasions when the event occurs.

If on-site weather measurements are unavailable, then weather data can be estimated from various approaches, such as spatial interpolation or nearest neighbour method (Kim et al. 2007). The performance of each approach can be summarised in a contingency table (Table 2), which shows the fraction of correct and incorrect prediction of a weather event. The prediction success is determined from weather measured at the site of interest, called the validation site.

TABLE 2: Contingency table for prediction and occurrence of a binary event. Note that $a+b+c+d=1$.

\begin{tabular}{lcc}
\hline & \multicolumn{2}{c}{ Observed } \\
\cline { 2 - 3 } Predicted & No & Yes \\
\hline No & $\mathrm{a}$ & $\mathrm{b}$ \\
Yes & $\mathrm{c}$ & $\mathrm{d}$ \\
\hline
\end{tabular}

Expected costs on a course of action that the decision maker has taken using a set of weather estimates $w$ are given by Richardson (2000):

$$
E_{w}=F_{w}(1-\bar{o}) X-H_{w} \bar{o}(G-X)+\bar{o} L
$$

where $\mathrm{H}=$ hit rate and $\mathrm{F}=$ false alarm rate. The hit and false alarm rates can be calculated using Table 2 as follows:

$$
H=d /(b+d)=d / \bar{o}
$$

and

$$
F=c /(a+c)=c /(1-\bar{o})
$$

The expected cost of using weather estimates would be greater than that of using weather measurements because of errors in weather estimates. The value of a method 
to estimate weather data can be defined as follows:

$$
V_{x, y}=\frac{E_{x}-E_{M}}{E_{y}-E_{M}}
$$

where $\mathrm{x}$ and $\mathrm{y}$ indicate estimation methods. Substituting from Eqs (1), (2), (3) and (4) into $\mathrm{Eq}(5)$, the relative value between two methods is:

$$
V_{x, y}=\frac{\bar{o} \cdot \alpha-F_{x} \cdot(1-\bar{o}) \cdot \alpha+H_{x} \cdot \bar{o} \cdot(1-\alpha)-\bar{o}}{\bar{o} \cdot \alpha-F_{y} \cdot(1-\bar{o}) \cdot \alpha+H_{y} \cdot \bar{o} \cdot(1-\alpha)-\bar{o}}
$$

where $\alpha$ indicates the 'cost-loss' ratio (X/G), which is the cost of taking action expressed as a fraction of the potential gain generated by the action (Richardson 2000). As a result, the relative value between two particular estimation methods for weather data becomes external to the system, because $V$ depends on $\alpha$ and $\overline{\boldsymbol{o}}$, and $\mathrm{H}$ and $\mathrm{F}$ are dependent on each estimation method. In addition, the 'return' ratio can be defined as the potential gain generated by the action in relation to the expense $(\mathrm{G} / \mathrm{X})$ and is the inverse of the cost-loss ratio. In practice, the value of $\mathrm{G}$ is dependent on the value of crops, which may vary season to season. Therefore, the cost-loss ratio is likely to be variable rather than constant. Thus, a grower can choose the better method using the relative value between two methods under a given condition.

The use of the cost-loss ratio model was simulated using a decision support system designed to minimise losses due to botrytis bunch rot on grapes. The risk of botrytis bunch rot can be determined from the value of an index calculated using weather data. Risk index values were obtained from the Bacchus model developed by B. Rengasamy and R. Edwards (Kim et al. 2007) from season-long weather data, using the implementation of Bacchus in the Metwatch programme (HortPlus ${ }^{\mathrm{TM}}$ Ltd). It was assumed that a grower takes action, e.g. fungicide spray application, when the accumulated risk value was greater than 4 in a week.

A simulation study was conducted for two validation sites, which were weather stations in the HortResearch horticultural weather station network, at Renwick (41 $30^{\prime} \mathrm{S}$ $173^{\circ} 51^{\prime} \mathrm{W}$ ) in Marlborough, and at Tomoana (39 37's $176^{\circ} 53^{\prime} \mathrm{W}$ ) in Hawke's Bay. Weather data measured at the validation sites (air temperature and surface wetness duration) between November and March in 2003-4 and 2004-5 were compared with weather data estimates. These estimates were measurements at the nearest neighbour station from the validation site (the nearest neighbour method) and weighted average of measurements at stations surrounding the validation site (spatial interpolation method) as described in Kim et al. (2007).

Estimated weather variables at the validation sites, using data from other nearby stations, were air temperature, vapour pressure and wind speed. These were used in an empirical model to estimate wetness duration (Kim et al.2007). Weather measurements and estimates were used as inputs to the Bacchus model to simulate the risk of botrytis bunch rot and a course of action that a grower would take. Instances where measured and estimated botrytis risk agreed represented correct grower decisions (a and d in Table 2) and instances where measured and estimated botrytis risk disagreed represented incorrect grower decisions (b and $\mathrm{c}$ in Table 2).

\section{RESULTS}

In total, the risk index value of botrytis bunch rot exceeded 4 in a week for 29 times at both sites during the study period. The frequency of occurrence of the event differed by site and season (Table 3 ). For example, the event occurred 3 times at Renwick in the 2003/4 season. In contrast, the event occurred 11 times at Tomoana in the same season. 
TABLE 3: The outcome of spay application simulation in 2003-4 and 2004-5 seasons.

\begin{tabular}{lccccc}
\hline Site & Season & $\mathrm{N}^{1}$ & $\overline{\boldsymbol{O}}^{2}$ & $\mathrm{H}^{3}$ & $\mathrm{~F}^{3}$ \\
\hline Nearest Neighbour & & & & & \\
Renwick & $2003-4$ & 24 & 0.13 & 0.67 & 0.00 \\
& $2004-5$ & 23 & 0.22 & 0.40 & 0.00 \\
Tomoana & $2003-4$ & 24 & 0.46 & 0.64 & 0.08 \\
& $2004-5$ & 23 & 0.43 & 1.00 & 0.08 \\
& & & & & \\
Spatial Interpolation & $2003-4$ & 24 & 0.13 & 1.00 & 0.05 \\
Renwick & $2004-5$ & 23 & 0.22 & 0.60 & 0.11 \\
& $2003-4$ & 24 & 0.46 & 0.91 & 0.31 \\
Tomoana & $2004-5$ & 23 & 0.43 & 1.00 & 0.23 \\
\hline
\end{tabular}

${ }^{1} \mathrm{~N}$ is the number of weeks in a season.

$2 \overline{\boldsymbol{O}}$ is the frequency of weeks in which spray application is advised using weather measurements as input to the Bacchus model.

${ }^{3} \mathrm{H}$ and $\mathrm{F}$ are the hit rate and the false alarm rate, respectively.

When weather estimates using spatial interpolation were used as inputs to the Bacchus model, the number of spray advisories was overestimated. In contrast, those using the nearest neighbour method resulted in underestimated spray advisories. Thus, the hit rate and false alarm rate of the spatial interpolation method were higher than those of the nearest neighbour method.

Overall, the value of spatial interpolation to estimate weather data was greater than that of the nearest neighbour method when the return ratio was $>2.6$ (Fig. 1). The relative value of spatial interpolation to the nearest neighbour method increased logarithmically with increasing return ratio $\left(\mathrm{R}^{2}=1.00\right)$. Weather estimates using the nearest neighbour method resulted in more valuable prediction only when the return ratio was relatively small, e.g. $<2.0$.

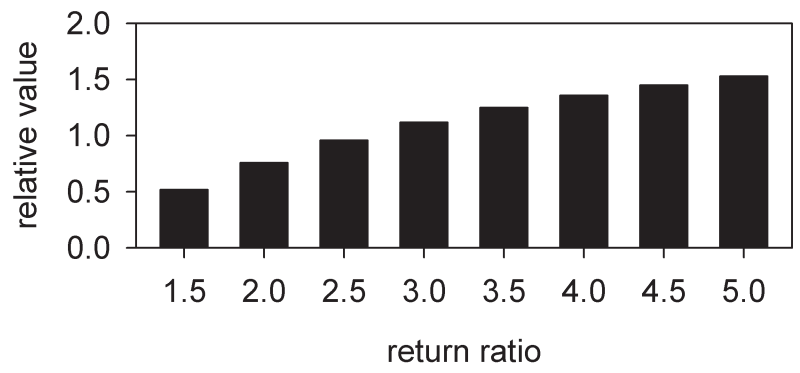

FIGURE 1: The relative value of spatial interpolation to estimate weather data in comparison with the nearest neighbour method. The value of each method was calculated simulating implementation of a decision support system for management of botrytis bunch rot on grapes. The 'return' ratio is the potential gain generated by an action in relation to the expense. 


\section{DISCUSSION}

These results showed that a cost-loss model was useful to choose an approach to estimate weather data, in order to operate a decision support system. In general, when different methods to estimate weather data are available to a decision maker, it might be assumed that the more accurate method weather estimation has more value (Kim et al. 2006). However, this fails to show that the relative value between them may be different under different circumstances, as the present study has suggested. For example, the nearest neighbour method was more useful when a low return was expected.

The advantage of using the cost-loss model is that it provides the economic value of weather estimates used as input to a disease warning systems. For example, Gleason et al. (1997) compared cumulative disease risk indices obtained from measured and estimated weather data to represent the value of weather estimates. Kim et al. (2006) analysed distribution of errors of spray application date predicted using weather estimates as the inputs to disease warning systems. However, these studies focused on determining accuracy in predicting occurrence of spray events rather than the economic value of weather data.

It is likely that spatial interpolation would provide more valuable data than the nearest neighbour method. When spray application is expected to be effective, weather estimates using spatial interpolation would incur less total cost than using the nearest neighbour method. Although spatial interpolation requires more computational resources than the nearest neighbour method, its use can be justified when high return for expended costs is expected. In addition, it is also important to make use of a disease risk prediction model that has high accuracy in predicting the risk of disease in order to predict spray application dates accurately.

This study focused on the use of hindcast estimates of weather variables using historical data from weather stations to provide information on management of botrytis bunch rot risk. In practice, weather forecasts are more useful than hindcast estimates because growers can prepare their spray application more effectively. For example, spray application can be limited by rainfall during a day on which spray application is advised using the hindcast estimates. Therefore, it would be useful to evaluate the value of methods that provide weather forecasts and this merits further study.

\section{ACKNOWLEDGEMENTS}

We thank B. Rengasamy and R. Edwards who developed the Bacchus model. This study was carried out with financial support from the HortResearch Capability Fund.

\section{REFERENCES}

Gleason ML 2000. Disease-warning systems. In: Encyclopedia of Plant Pathology. Volume I. John Wiley \& Sons, New York. Pp. 367-370.

Gleason ML, Parker SK, Pitblado, RE, Latin RX, Speranzini D, Hazzard RV, Maletta MJ, Cowgill WP Jr., Biederstedt DL 1997. Validation of a commercial system for remote estimation of wetness duration. Plant Disease 81: 825-829.

Kim KS, Beresford RM, Henshall WR 2007. Prediction of disease risk using site-specific estimates of weather variables. New Zealand Plant Protection 60: 128-132.

Kim KS, Taylor SE, Gleason ML 2006. Forecasting site-specific leaf wetness duration using empirical models. Plant Disease 90: 650-656.

Meyers DE 1994. Spatial interpolation: An overview. Geoderma 62: 17-28.

Richardson DS 2000. Skill and relative economic value of the ECMWF ensemble prediction system. Quarterly Journal of the Royal Meteorological Society 126: 649-667. 